Relmecs, diciembre 2017, vol. 7, no. 2, e030, ISSN 1853-7863

Universidad Nacional de La Plata. Facultad de Humanidades y Ciencias de la Educación. Centro Interdisciplinario de Metodología de las Ciencias Sociales.

Red Latinoamericana de Metodología de las Ciencias Sociales

\title{
Mundo plural: ipor qué los individuos hacen lo que hacen?
}

\author{
Bernard Lahire * \\ * Instituto Universitario de Francia, Francia | bernard.lahire@ens-lyon
}

\section{PALABRAS CLAVE RESUMEN}

Teoría Social En un contexto de hiper-especialización, el artículo se pregunta por una visión panorámica de la sociedad y una concepción compleja de los individuos, que no olvide a la vez la ambición teórica y el pulso empírico.

Investigación empírica Con este objetivo, se pregunta: “¿por qué los individuos hacen lo que hacen?” y propone responder con una fórmula de estudio de las prácticas de carácter universal que combina una dimensión disposicionalista con

Prácticas una contextualista. De un lado, las disposiciones y competencias de los individuos, entendidas como el producto interiorizado de la frecuentación más o menos intensa a determinados contextos de acción. Del otro

Disposiciones lado, los contextos de la acción presente y su diversidad. Desde esta fórmula, el artículo evalúa distintas

Contextos aproximaciones a lo social: por un lado, el olvido del pasado, característico de las sociologías pragmatistas, interaccionistas y de la elección racional; por el otro lado, el olvido de los contextos, típico de las sociologías bourdieanas y de ciertas teorías psicoanalíticas. Para finalizar, el artículo repasa la diversidad de las investigaciones contemporáneas en ciencias sociales (en relación con la escala de la observación, el nivel de la realidad social estudiado, el interés de conocimiento, el tipo de objeto, las escalas temporales, el número de actores estudiados, etc.), mostrando que el enfoque disposicional-contextualista puede orientar trabajos en dichas direcciones tan diferentes.

\section{KEYWORDS ABSTRACT}

Social Theory

In a context of hyper-specialization, the article addresses a panoramic view of society and a complex conception of individuals, while not forgetting both the theoretical ambition and the empirical pulse. To this end, it asks: "why do individuals do what they do?", and proposes to respond with a universal formula for the study of practices that combines a dispositional dimension with a contextualist one. On the one hand, the

Empirical Research

Practices dispositions and competences of individuals, understood as the internalized product of more or less frequent access to certain contexts of action. On the other hand, present contexts of action and their diversity. From

Dispositions

Contexts this formula, the article evaluates different approaches to the social: on the one hand, the oblivion of the past, characteristic of pragmatist, interactionist and rational choice sociologies; on the other hand, the neglect of contexts, typical of certain uses of Bourdieu's sociology and certain psychoanalytic theories. Finally, the article reviews the diversity of contemporary research in social sciences (in relation to the scale of observation, the level of social reality studied, the interest in knowledge, the type of object, the time scales, the number of actors studied, etc.), showing that the dispositional-contextualist approach can guide works in such different directions. 
La sensación de dispersión entre los trabajos de las ciencias humanas y sociales es el producto de la gran división social del trabajo científico en disciplinas separadas (con ciencias de la "psique”, ciencias del "lenguaje”, ciencias de la "sociedad", ciencias de la "economía”, ciencias de lo "político", etc.) y en sectores especializados en el seno de cada disciplina (sociología de la educación, de la familia, de la cultura, del trabajo, del deporte, etc.).

Una hiper-especialización científica tal, que lleva a distintos investigadores a estudiar por separado cada área de prácticas, cada sector de la vida social, y a formular teorías parciales del actor, no hace más que acompañar ciegamente el largo proceso histórico de diferenciación social de las actividades. Presos en este movimiento de diferenciación que caracteriza a las sociedades modernas, los eruditos son cada vez menos capaces de estudiar sus efectos.

¿Cómo se puede hacer una panorámica del conjunto del mundo social cuando todo empuja a cada categoría de investigadores a mantener la nariz pegada sobre el funcionamiento de pequeñas parcelas de este mundo? ¿Cómo mantener una concepción compleja de los individuos en sociedad cuando, en primer lugar, los recortes disciplinarios, luego las especializaciones internas, fuerzan a los investigadores a trabajar sobre dimensiones cada vez más específicas de las prácticas individuales? ¿Cómo conservar un alto grado de creatividad científica cuando una concepción estrecha del profesionalismo conduce insensiblemente hacia una especialización extrema de los investigadores?

Responder a este conjunto de preguntas, es abrazar lo que está en juego en las ciencias humanas y sociales y sus desafíos, intentando recuperar las grandes ambiciones científicas originales - especialmente las de Durkheim o de Weber- y evitando a la vez la regresión hacia las formas empíricamente perezosas y teóricamente pretenciosas de pensamiento de la "totalidad" o de la "complejidad".

Hacer el duelo de la "gran teoría social" o de la "teoría general social" no implica abandonar todo programa científico ambicioso. Asumir el desafío de una ambición tal exige, sin embargo, proponer respuestas adaptadas al estado problemático de las ciencias humanas y sociales existentes. Hay que reconocer sobre todo que el programa científico en cuestión sólo pueda dar lugar a logros empíricos frecuentemente imperfectos y parciales. Pero los diferentes trabajos empíricos existentes no tienen el mismo sentido según se los presente como trabajos totales y completos en su estilo, o si son concebidos y leídos como realizaciones particulares de tal o cual parte de un programa científico general.

Este programa, que responde a la pregunta de saber por qué los individuos actúan como actúan, piensan como piensan, sienten como sienten, etc., puede resumirse en una fórmula científica bastante simple:

Pasado incorporado+Contexto presente de acción = Prácticas .

Esta última condensa la intención de la investigación que consiste en pensar las prácticas en el cruce de las disposiciones y competencias incorporadas (producto de la frecuentación más o menos duradera de los marcos socializadores pasados) y del contexto siempre específico de la acción. Sin embargo, constatamos desgraciadamente que la sensación de dispersión de los trabajos de las ciencias humanas y sociales se amplifica por la desarticulación de esta fórmula de la cual sólo se estudian partes, aun pretendiendo alcanzar la explicación más completa posible.

Comprender porqué individuos particulares, o grupos sociales, grandes o pequeños, hacen lo que hacen, piensan lo que piensan, sienten lo que sienten, dicen lo que dicen, he aquí condensado en algunas palabras el ambicioso objetivo de las ciencias humanas y sociales - sociología, historia, antropología, geografía, etc-. Este objetivo, me parece que estas ciencias lo alcanzan con mayor precisión y mayor pertinencia cuando recogen las prácticas en el cruce de las propiedades sociales de los actores y de las propiedades sociales de los contextos en los cuales inscriben sus acciones.

Y si se quiere llevar la reflexión teórica un poco más allá y darse los medios para pensar más conscientemente y 
más sistemáticamente la articulación entre los actores y los contextos, es necesario nombrar y describir las direcciones de la investigación que sustentan la operación de articulación en cuestión.

\section{Las prácticas entre disposiciones y contextos}

En estas circunstancias, cualquier investigador que se esfuerza, en investigaciones empíricas determinadas, por alcanzar el punto de equilibrio explicativo entre, por un lado, el estudio de las propiedades sociales incorporadas de los actores, y, por el otro, el de las propiedades sociales objetivadas de los contextos, combina inevitablemente un disposicionalismo y un contextualismo. Comprender las prácticas o los comportamientos a través de una reconstrucción de los tipos de disposiciones mentales y comportamentales incoporados que los actores llevan consigo (producto de la interiorización de experiencias sociales pasadas) y de las características de los contextos particulares (naturaleza del grupo, de la institución o de la esfera de actividad, tipo de interacción o de relación) en los que evolucionan es, a mi entender, la vía científicamente más rentable que los investigadores están en condiciones de poner en práctica.

Que las realizaciones empíricas de los que adhieren a este programa sean siempre imperfectas y sólo alcancen excepcionalmente el punto de equilibrio perfecto - por razones que remiten a menudo tanto a cuestiones prosaicas de tiempo limitado de la investigación, como de acceso restringido a ciertos datos empíricos o de obstáculos sociales diversos en la puesta en práctica de métodos, más que a falta de buena voluntad o de seriedad de los investigadores - no debería cuestionar la validez general del modelo teórico disposicionalista-contextualista.

De cualquier modo, esto no justifica en absoluto el espectáculo que nos presentan a menudo las "comunidades eruditas”, es decir el recorte de los diferentes elementos del problema que se busca resolver en una cantidad de posiciones teóricas (y hasta a veces disciplinares) separadas, opuestas unas a otras.

Este estado de situación no responde a lógicas intrínsecamente científicas, sino más bien a lógicas de competencias y de "necesidades" de existir distintivamente, y sobre todo de distinguirse de la manera más rápida y más estruendosa posible. Cuando un campo teórico parece dominar temporalmente, no hay que esperar mucho para ver aparecer un campo opuesto, que, más que intentar apropiarse de los logros de los trabajos realizados, prefiere reivindicar una posición radicalmente nueva. La radicalidad siendo la condición de visibilidad más nítida, todos los que buscan más ser visibles que resolver problemas científicos tienen un interés en cierto radicalismo teórico.

Comenzar por incorporar los logros de los trabajos de los cuales de hecho se busca diferenciarse en una serie de cuestiones, es tomar el riesgo de no llamar la atención y de no hacerse notar lo suficiente. Es por esto que numerosos autores se "cambian de bando". De este modo, los intelectuales demuestran más su dependencia de sus competidores que su preocupación por ir hacia un poco más de verdad, de sutileza y de complejidad. Ya habrán entendido que, dado como funcionan socialmente los universos intelectuales, los que se cambian de bando tienen todavía un bello porvenir por delante.

Pero volvamos a lo fundamental. Para resumir el enfoque científico indisociablemente disposicionalista y contextualista, podemos enunciar la siguiente fórmula:

\section{Disposiciones + Contextos $=$ Prácticas}

Las prácticas consideradas (ya sea una “elección” alimentaria o de vestimenta, deportiva o política, un comportamiento escolar o económico, sexual o cultural, profesional o familiar, etc.) sólo se comprenden entonces si se estudia, por un lado, las exigencias contextuales que pesan sobre la acción (lo que el contexto exige o solicita a los actores) y, por otro, las disposiciones socialmente constituidas a partir de las cuales los actores perciben y se 
representan la situación, y sobre la base de las cuales actúan en esta situación. En esta fórmula, nos damos cuenta de que, si las prácticas pueden observarse y registrarse en tanto realidades presentes y, que si los contextos de acción son objetivables por el investigador al considerar las "reglas" de su juego, las especificidades de su funcionamiento y la naturaleza de las relaciones que allí se desarrollan (que hacen que el contexto escolar se diferencie del contexto religioso o político, pero también que el micro-contexto escolar del aula se distinga del micro-contexto escolar del patio, o que el micro-contexto de la clase de lengua se diferencie del micro-contexto de la clase de plástica), las disposiciones, en cambio, no se observan directamente y remiten al pasado de los actores estudiados. La misma fórmula puede, por lo tanto, ser presentada de la siguiente manera:

Pasado incorporado + Contexto presente $=$ Prácticas observables.

\section{Socializaciones y pasados incorporados}

En relación a la primer parte de la fórmula - el "pasado incorporado"- se trata de todo lo que el actor importa a la escena de la acción, de todo lo que le debe al conjunto de las experiencias que ha vivido y que se han cristalizado en él bajo la forma de capacidades y de disposiciones para actuar, sentir, creer, pensar más o menos fuertes y permanentes. Los investigadores califican los procesos de fabricación de las disposiciones y de las competencias (capacidades) como procesos de socialización y hablan de experiencias socializadoras cuando quieren poner el acento sobre lo que los contextos de acción vividos “imprimen” en tanto cambio, modificación, transformación sobre los actores. El pasado incorporado es entonces únicamente el efecto de la frecuentación pasada - más o menos precoz, duradera, sistemática- de los diversos contextos de acción (familiar, escolar, profesional, religioso, político, cultural, deportivo, etc.):

Productos interiorizados de la frecuentación pasada de contextos de acción

+ Contexto presente $=$ Prácticas observables

Esto significa que el contexto presente de la acción puede ser considerado desde dos puntos de vista distintos:

- como marco desencadenante de disposiciones incorporadas,

- o como marco socializador de los actores.

Cuando los actores en cuestión son niños, vemos inmediatamente en qué medida los contextos de acción son también contextos de socialización, por ende marcos en los cuales se forman competencias, apetencias o hábitos mentales y comportamentales. Los investigadores en ciencias sociales piensan, desgraciadamente, rara vez en actores-niños. Pero mismo cuando se trata de adolescentes o de adultos, la frecuentación repetida de ciertos contextos de acción nunca se hace sin consecuencias socializadoras (o, dicho de otro modo, disposicionales) sobre ellos.

Ya sea que hablen de "disposiciones”, de "hábitos", de "inclinaciones", de "tendencias", de "debilidades”, de “propensiones”, de "capacidades”, de "competencias”, de "trazos mnésicos”, de "esquemas”, de "ethos” o de "habitus", los investigadores se esfuerzan por tener en cuenta el hecho científico evidente -de un punto de vista sociológico como neurocientífico- que constituye la incorporación por los hombres de los productos de sus experiencias sociales. El Hombre es un animal social, en el sentido en que está hecho biológicamente - con el cerebro y el sistema nervioso de los cuales está dotado - para memorizar, conservar o cristalizar los productos de sus experiencias, ya sean explícitamente o no dirigidas hacia el aprendizaje de saberes.

Hay que tomar muy en serio la intuición de Jean-Pierre Changeux según la cual los conceptos disposicionalistas que suponen que nuestros aprendizajes o nuestras experiencias se inscriben bajo la forma de trazos más o menos 
duraderos en el cerebro - permiten relacionar a las neurociencias con las ciencias humanas y sociales $\underline{2}$. Si los seres humanos no fuesen capaces - biológicamente - de "memorización” (en el sentido de incorporación noconsciente como de memorización por un esfuerzo conscientemente realizado), las ciencias humanas y sociales sólo requerirían un programa contextualista y podrían contentarse con ser ciencias de los contextos sociales. Nuestras conductas serían explicables a partir del momento en que seríamos capaces de precisar los contextos en los cuales actuamos. Según el tipo de conducta o de práctica estudiada y según el grado de precisión analítica buscado, los investigadores establecerían que el marco de interacción o el área de la práctica, el lugar en la organización o en la institución, la posición en el campo o en el subsistema social determinan todos los comportamientos observables. Si fuese así, la interpretación en las ciencias humanas y sociales sería infinitamente menos compleja de lo que es en realidad.

En primer lugar, las experiencias sociales relativamente análogas que se repiten pueden cristalizarse bajo la forma de capacidades o de competencias para realizar ciertas cosas. Las capacidades o competencias sólo son potencialmente movilizables y sólo son realmente movilizadas por los actores cuando las situaciones lo exigen. Por ejemplo, las capacidades para calcular mentalmente, las competencias culinarias, las competencias del jugador de cartas o del bailarín de tango, las capacidades para descifrar oralmente un texto escrito o para conducir un auto, son algunos saberes y savoir-faire adquiridos (disponibles en el patrimonio de las competencias de los individuos), que esperan las situaciones de su despliegue. Si se entrena regularmente a hacer malabares o a andar en bicicleta, las capacidades quedan intactas y hasta pueden mejorarse, o complejizarse, cuando se pasa por ejemplo de hacer malabares con dos pelotas a hacerlo con tres. Pero un abandono o la baja de intensidad de la práctica también torna estas capacidades más inciertas, menos potentes $\underline{3}$.

En una presentación abreviada de su famoso Tratado sobre la naturaleza humana, el filósofo David Hume subraya la importancia de nuestras experiencias que, al repetirse, crean hábitos mentales y funcionan, en el transcurso de la práctica, como anticipaciones prereflexivas de fenómenos futuros $\underline{4}$. Hume toma de este modo el ejemplo de la interacción entre dos bolas de billar. La primera, que está en movimiento, impacta contra la segunda, inmóvil, que, bajo el efecto del choque, se pone en movimiento a su vez. Quien haya tenido la experiencia de este tipo de situación puede constatar cada vez el mismo fenómeno (Hume habla de "conjunción constante entre la causa y el efecto") y termina, de este modo, en situaciones análogas, por anticipar los efectos producidos (el choque, luego la puesta en movimiento de la bola inicialmente inmóvil): "Supongan que veo una bola desplazarse en línea recta hacia otra; concluyo inmediatamente que van a chocarse y que la segunda va a ponerse en movimiento.”

Para Hume, es sobre la base de una experiencia repetida que una disposición mental se formó -por habituaciónpoco a poco. Y es esta disposición la que conduce al individuo que percibe y actúa a proyectar en el futuro (y por ende a anticipar acciones que aun no tuvieron lugar) el producto interiorizado de sus experiencias pasadas:

"Estamos determinados solamente por HABITUACION a suponer que el futuro será acorde al pasado. Cuando veo una bola de billar moverse hacia otra, mi mente es llevada inmediatamente por hábito al efecto usual, y ella se anticipa a mi vista concibiendo la segunda bola en movimiento."

Hume nos brinda aquí el modelo de cualquier teoría disposicionalista de la acción, la que, teniendo en cuenta los productos incorporados de las series de experiencias pasadas relativamente análogas, toma conocimiento del hecho de que el presente de la acción o de la interacción, y sobre todo las propiedades del contexto de acción, no lo explican todo acerca del comportamiento de los actores. Resalta también el hecho de que la "razón” y la "voluntad" intervienen poco en las representaciones ordinarias que, fundadas en creencias prácticas adquiridas por experiencia, id. est. por habituación a situaciones relativamente similares, constituyen más bien hábitos prereflexivos que esquemas explícitos y conscientes, intencionalmente puestos en marcha:

“No es entonces la razón el guía de la vida, sino la habituación. Sólo ella determina que la mente, en todos los casos, suponga que el futuro será acorde al pasado”. 
Basta con reemplazar el ejemplo de las bolas de billar en interacción sobre una mesa por situaciones propiamente sociales, y variables según la época o el grupo, para darse cuenta de la importancia de una reflexión tal para la ciencias humanas y sociales. Pensaremos, por ejemplo, en el modo en el que un individuo -niño, adolescente o adulto- se vuelve sensible a ciertos juegos del lenguaje, a ciertas formas de relaciones sociales, a ciertos modos de ejercicio de la autoridad, a ciertos modos de razonamiento o a ciertos tipos de comportamientos o actitudes morales, culturales, estéticas, políticas, etc. Frente a tal o cual situación, el actor acciona o reacciona en función de lo que cree reconocer inmediatamente de los imperativos ligados a esta situación en función de sus experiencias pasadas. Lo que el actor percibe, ve, siente o se representa de la situación presente y lo que hace al respecto sólo se comprende en el cruce de las propiedades (objetivables) de la situación en cuestión y de sus propiedades incorporadas (disposiciones mentales y comportamentales más o menos coherentes o contradictorias formadas en el curso de experiencias socializadoras pasadas). El accionar presente está asediado por la memoria involuntaria de la experiencia pasada.

Tendencias a actuar, maneras de hacer, creencias, esquemas de percepción y de representación, disposiciones y competencias (o capacidades) no son reductibles a "conocimientos", a "cuadros de interpretación" o a "visiones del mundo". Son tan comportamentales como mentales (Marcel Mauss distinguía "hábito práctico” y "hábito mental”든 y es por esta razón que el disposicionalismo se distingue de las concepciones que, aun tomando en cuenta los procesos de interiorización del mundo social, reducen estos procesos al aprendizaje de conocimientos y de significados.

Si queremos terminantemente tender a una cierta "universalidad" en las ciencias humanas y sociales, ciertamente no es en el orden de "reglas" improbables o "valores" que existen pretendidamente en todas las sociedades humanas que hay que buscarla. En cambio, en todas las sociedades humanas, hay grupos, formas de vida y de actividad colectivas e individuos que son socializados y que actúan en estos grupos o estas formas de vida colectivas. En todas las sociedades humanas se articulan disposiciones y competencias (productos de la frecuentación más o menos duradera de las diferentes formas de vida social y, a la vez, productos de una socialización implícita o de aprendizajes intencionales) y “contextos de acción”, cuya naturaleza varía según el tipo de sociedad y en el seno mismo de una determinada sociedad. La "universalidad” de una articulación tal no está exenta de relación con las capacidades naturales, biológicas del hombre, con sus capacidades mnemónicas, el tipo de cerebro del que dispone y que lo diferencia de otros animales.

\section{El olvido del pasado}

La fórmula que articula pasados incorporados y contextos presentes de acción debería, en mi opinión, tener un rol estructurante en las ciencias humanas y sociales. Sin embargo, a pesar de la evidencia disposicionalista, algunas teorías de la acción hacen como si los actores fuesen "sin pasado", totalmente amnésicos, y enteramente maleables bajo el efecto de obligaciones que pesan sobre los diferentes contextos de acción. Estas teorías se interesan menos por los actores accionando que por la acción en sí, sea cual sea la historia de los actores. Presuponen entonces o bien que es posible hacer un análisis del mundo social sin pasar por el estudio de los actores, o que los actores son adultos definitivamente constituidos y que nunca fueron niños.

Fuera el estudio de los procesos de socialización, de los procesos de construcción de la memoria (de las memorias) o de los hábitos mentales y comportamentales. Teoría de los sistema sociales que excluyen a los actores, estudio del “orden de interacción” independientemente de las experiencias pasadas de los actores, estudio formal de las organizaciones, de las instituciones o de los sistemas de acción que se contentan con tomar en cuenta la posición de actores despojados de todo pasado, teorías de la acción racional, que dotan a los actores (homo œconomicus o actor calculador y estratega) de una psicología universal superficial, o sociologías dichas “pragmáticas” que les atribuyen una serie de competencias sin historia $\underline{6}$, todas ellas contribuyen a desequilibrar la fórmula científica quitándole uno de sus términos: 
Pasado incorporado + Contexto presente $=$ Prácticas.

En algunos trabajos etnográficos, los investigadores pueden considerar a veces que terminaron su trabajo de análisis una vez que describieron precisamente una situación o el curso de una acción, o bien, luego de la descripción, una vez que extrajeron algo parecido a una regularidad (reglas de conversación, una gramática de la acción, estructuras invariantes de la relación o de la actividad). Pero se topan con un hecho ineludible, es decir que no se puede verdaderamente comprender porqué los individuos reaccionan de modo distinto $-\mathrm{y}$ a veces contradictorio - ante los mismos "estímulos" exteriores, los mismos contextos, si se olvida que, siendo caracterizados por una serie de experiencias pasadas diferentes, "entran” en un contexto con el peso de todo un pasado incorporado, sedimentado de maneras más o menos durables de ver, de sentir y de actuar.

Pero los investigadores atentos al peso del pasado incorporado, o explícitamente inscriptos en una tradición disposicionalista, en el estudio de los hechos sociales, descuidan, a veces ellos también, los efectos de este pasado incorporado. De este modo, por ejemplo, aunque habla de "habitus" y evoca a menudo los procesos de interiorización de las obligaciones sociales, Norbert Elias pone en práctica un pensamiento configuracional, que insiste más sobre las relaciones de interdependencia que forman entre ellos los individuos y sobre las obligaciones que éstas hacen pender sobre los comportamientos de cada uno de ellos, que sobre la articulación de estos “paralelogramos de fuerzas” y del pasado incorporado por individuos socializados $\underline{7}$.

Un deslizamientocontextualista similar se observa también del lado de los trabajos hechos a partir de la teoría de los campos, que terminan ligando el conjunto de las prácticas y representaciones de cada agente a su posición en el campo, reduciendo a casi nada las características y efectos del habitus del que ocupa esta posición.

\section{El olvido de los contextos}

Si el pasado es a menudo negado o marginalizado por una parte de los investigadores, podemos igualmente insistir, a la inversa, en las derivas de los disposicionalistas, que tienden a descuidar la especificidad y la variación de los contextos de acción, con todos los efectos de transformación de los comportamientos que esta variación implica. Es el caso a veces de la teoría del habitus que hace del habitus un principio de conservación y, por ende, de selección de los contextos frecuentados, de refugio de las crisis disposicionales, olvidando todos los contextos que no están siempre sometidos a la "elección" o al "deseo" de los actores, sino que se imponen a ellos con una fuerza coercitiva: universo escolar, marco profesional, institución hospitalaria, situación política, religiosa, situaciones de dominación colonial, de guerra, de encierro, etc.

Es también el caso de las diferentes variantes de la teoría psicoanalítica que, centradas en las experiencias familiares de la primera infancia, hacen a veces de toda vida adulta la historia del eterno retorno de los esquemas de la experiencia originalmente construidos. En todo caso, el peso o el rol del contexto es tan descuidado que se lo reduce a ser sólo la superficie de actualización de los mismos esquemas mentales y comportamentales, el terreno en donde se representan permanentemente las mismas escenas, la ocasión de un despliegue de las mismas lógicas. También allí, la fórmula científica se desequilibra, a favor esta vez de las experiencias socializadoras incorporadas:

Pasado incorporado + Contexto presente $=$ Prácticas .

Todo parece estar jugado de antemano para los actores mismo antes de que hayan tenido la oportunidad de entrar en los diferentes juegos que se les presentan.

Se presupone entonces la transferibilidad o la extrapolabilidad de las disposiciones (esquemas, hábitos, etc.) y se 
representa la realidad de las disposiciones o de las estructuras psíquicas como una realidad relativamente homogénea, cuya coherencia está asegurada ya sea por las condiciones sociales de existencia características del medio familiar (teoría del habitus), ya sea por las características (supuestas más o menos universales) de la configuración familiar compuesta por el Padre, la Madre y los hijos.

A esto, podemos oponerle la comprobación de la pluralidad de las disposiciones y competencias de las que son más o menos portadores los actores sociales y los hechos de no-transferibilidad de las disposiciones incorporadas $\underline{8}$. Cuanto más hayan los actores frecuentado contextos sociales (y socializadores) heterogéneos, y cuanto más precoz haya sido esta frecuentación, en el seno de la configuración familiar (sobre todo por el hecho de la diferencia social entre sus miembros) o por el hecho de la diversidad de los contextos socializadores (familia, escuela, guardería, niñeras o cualesquiera otros agentes o instituciones socializadoras), más serán éstos portadores de disposiciones heterogéneas y a veces contradictorias.

Las disposiciones no actúan de manera permanente, sino solamente en función de los contextos de acción que se presentan. No se trata entonces de una actualización sistemática de las mismas disposiciones (del mismo sistema de disposiciones o de la misma fórmula generadora de prácticas), pero de un juego más complejo de activación y de inhibición de las diferentes disposiciones incorporadas que pueden combinarse parcialmente entre ellas en determinadas situaciones, o funcionar a veces independientemente unas de otras en otras situaciones. En cualquier caso, si existen disposiciones permanentes (trans-contextuales), no son todas de este orden.

\section{El habitus y el campo como casos particulares entre otros}

Todas estas derivas hacia análisis unilaterales, exclusivamente contextualistas o exclusivamente disposicionalistas, prueban que es muy difícil para las ciencias humanas y sociales sostener el equilibrio de la fórmula científica en el análisis de las prácticas sociales. Pero el equilibrio es aun más difícil de sostener cuando no se explicita la fórmula en cuestión. La apuesta que hago, esclareciendo el trabajo de interpretación, es justamente dar la posibilidad de tender lo más que se pueda hacia el equilibrio.

Cualquiera que conozca bien la obra de Pierre Bourdieu habrá quizá reconocido en la fórmula:

“Disposiciones (y competencias) + Contexto = Práctica”

una declinación de la ecuación que el sociólogo enunciaba en 1979:

$$
\text { “[(habitus) }(\text { capital) }]+\text { campo = práctica”모 } .
$$

Aunque comparto los grandes principios, y sobre todo el equilibrio explicativo que inscribe como exigencia central en la práctica de la investigación, me diferencio en una serie de cuestiones que tienen que ver con los términos mismos de la ecuación: habitus, campo y práctica.

En primer lugar, el habitus como "sistema de disposiciones duraderas y extrapolables" sólo es uno de los casos posibles, un caso particular en el conjunto de los patrimonios individuales de disposiciones y competencias observables. No se puede suponer, como lo hace Pierre Bourdieu, que todas las disposiciones son "permanentes" y, por ende, de igual fuerza y durabilidad. Todo depende aquí del tiempo de socialización: de la mayor o menor precocidad de las experiencias socializadoras, del grado de intensidad con el cual la disposición ha sido formada, estabilizada y mantenida, y, por último, de la mayor o menor duración de formación y de fortalecimiento de la disposición. Todas las disposiciones para creer, para actuar, para sentir, para pensar de una cierta manera no gozaron de las mismas condiciones de socialización y no pueden tener, por ende, la misma fuerza, el mismo grado 
de permanencia y la misma capacidad para transferirse de un contexto a otro. Son desigualmente fuertes y hasta pueden, si no encuentran las condiciones para su actualización, debilitarse.

La misma idea de habitus como "principio generador y unificador" de los comportamientos, como "fórmula generadora de las prácticas”, que permite pensar “de manera unitaria” las diferentes dimensiones de la práctica de un individuo o de una clase de individuos dados, trae problemas en la medida en que da la impresión de que el conjunto de las disposiciones incorporadas por una persona en el curso de su existencia conforma un todo, un sistema completo que funciona "como un único hombre”, en el seno del cual el conjunto de las disposiciones son solidarias unas de otras y se funden en un único principio. La realidad de los patrimonios individuales de disposiciones y competencias, cuando nos tomamos el trabajo de estudiarla de cerca, es totalmente otra:

- las competencias a la espera de movilización existen tanto como las inclinaciones irreprimibles;

- las disposiciones débiles, que requieren contextos de mucha presión o muy favorables para actualizarse, se mezclan con las disposiciones fuertes que pueden ser puestas en marcha, independientemente de toda voluntad, aun en los contextos más inadecuados;

- las disposiciones específicas, que se transfieren bastante mal y están atadas a contextos sociales muy particulares, se distinguen de las disposiciones generales e intransferibles;

- las disposiciones se activan o se ponen en suspensión (están objetivamente impedidas, inhibidas y hasta a veces explícitamente desaconsejadas y sancionadas) en función de los contextos que se presentan;

- las disposiciones se combinan distinto entre sí según los contextos de práctica;

- disposiciones heterogéneas, hasta contradictorias, cohabitan en el seno del mismo patrimonio individual de disposiciones, etc.

En la escala individual, el estudio de los patrimonios de disposiciones y de competencias encuentra el carácter polifónico y a veces conflictivo que el psicoanálisis puso en evidencia acerca del psiquismo. Este último está la más de las veces constituido por conflictos internos entre tendencias opuestas y Freud lo concebía como una suerte de micro-sociedad en donde se enfrentan tendencias contrarias.

Si se puede pensar evidentemente en las distancias entre los deseos socialmente constituidos y las normas morales interiorizadas que los inhiben o los reprimen, también están todas las tensiones o las contradicciones posibles entre los diferentes miembros de una familia, particularmente entre el padre y la madre, entre lo que dicen los padres y lo que hacen, entre lo que quieren "transmitir" explícitamente y las condiciones en las que se desarrollan las "transmisiones", etc. La diversidad de los tipos de disposiciones y de competencias (corporales, estéticas, culturales, escolares, religiosas, morales, económicas, políticas, etc.), las múltiples diferencias y las posibles contradicciones entre las disposiciones para creer (modelos soñados, normas o ideales perseguidos) y las disposiciones para actuar, todo esto hace del modelo del "habitus" una suerte de primer prototipo teórico que permitió emprender investigaciones en una dirección prometedora, pero que no resiste -si tomamos en serio su definición más precisa y científicamente exigente - al examen de una serie de hechos.

La obra sobre la cual se apoya esta conferencia está enteramente dirigida hacia el concepto de "contexto de acción” que entra en la ecuación formulada anteriormente: 
Disposiciones + Contextos $=$ Prácticas

Intenté mostrar en este libro que cualquier contexto pertinente no es necesariamente un "campo", ni tampoco un “mundo", en el sentido de Howard Becker o de Anselm Strauss, y que es, además, útil distinguir diferentes tipos de "campos" (por ejemplolos "juegos" como "campos secundarios"). Se progresa científicamente a menudo cuando se logra mostrar que un concepto que se presenta bajo la forma de un concepto general, como el de habitus o el de campo, sólo es un caso particular de un fenómeno más general.

De este modo ya procedí acerca del hábito, mostrando que sólo era un caso entre otros en el conjunto real de los patrimonios individuales de disposiciones y de competencias. Y es de este mismo modo, integrador, que procedo en relación al campo, que es sólo un tipo de microcosmos estudiable en el conjunto de los universos socialmente diferenciados en el seno de sociedades altamente diferenciadas.

\section{La variación de los encuadres}

Además, es crucial interrogarse acerca de los otros encuadres posibles de los hechos o realidades sociales. El mismo hecho puede ser considerado en la perspectiva de una teoría de los campos y serlo en la perspectiva de cualquier otra teoría. En los diferentes casos, no se insistirá exactamente en la misma dimensión de los fenómenos y no se tirará de los mismos hilos. Las discusiones científicas pueden tener entonces como objetivo fijar qué teoría permite mejor resolver un tipo particular de problema, pero nunca zanjar definitivamente la cuestión de la "teoría verdadera" del mundo social.

Diciendo esto, no quiero defender ningún tipo de escepticismo o de relativismo científicos. Todos los trabajos no están al mismo nivel y una cierta acumulatividad crítica se desarrolló desde los trabajos de los grandes fundadores: los grados variables de robustez lógica de la argumentación, de fineza en la interpretación y de severidad empírica crean suficientes diferencias entre los trabajos más débiles y las obras consideradas más fuertes, y que son más resistentes al tiempo $\underline{10}$. Pero es necesario constatar, que, consideradas desde el punto de vista de los principios que los guían y de los objetivos que persiguen, los diferentes modelos teóricos de construcción de los objetos pueden difícilmente ser considerados como respuestas diferentes a las mismas preguntas o como soluciones alternativas a los mismos problemas.

Los investigadores deberían tener en cuenta en su discusión sobre los contextos, no solamente:

1) la escala de observación del mundo social: desde el estudio de acciones o de interacciones de cortas duraciones al estudio de datos cuantitativos vastos que permiten comparar sociedades, de grupos o de categorías, pasando por el análisis de trayectorias individuales, pero también

2) el nivel de realidad social para el cual pretenden ubicar su aporte de conocimiento: una observación hecha a una escala muy micro puede concebirse perfectamente en vistas de contribuir al conocimiento de grandes fenómenos macrosociológicos o macrohistóricos o, al contrario, ser conducida en la óptica de un mejor conocimiento de micro-procesos,

3) el interés por el conocimiento en juego que hace que, aunque compartan el mismo nivel de realidad social buscado, las teorías de los campos y de los mundos no observan realmente las mismas realidades, y, por ende,

4) el tipo de objeto (de "práctica" o de "hecho") que se dan como objetivo interpretar: el investigador no movilizará los mismos contextos ni los mismos elementos del pasado incorporado para interpretar la elección de una profesión, la interacción repentina y fuera del marco institucional entre dos completos desconocidos, la práctica de un deporte o la naturaleza particular de la obra literaria de un determinado escritor.

Lo que sorprende al observador de los trabajos contemporáneos de las ciencias humanas y sociales, y particularmente de los trabajos llamados "sociológicos", es la extrema diversidad de los tipos de objetos 
estudiados: desde el estudio de interacciones de corta duración o de recorridos biográficos individuales al análisis comparado de grupos sociales o de clases sociales a la escala de un espacio social nacional (con la pregunta central por la desigualdad), pasando por el estudio de prácticas o de la sociogénesis de grupos, de instituciones o de medios particulares.

En el conjunto de los trabajos existentes, se constata:

- una variación de la escala de observación adoptada,

- una variación del nivel de realidad social sobre el cual se pretende hacer abarcar el conocimiento: algunos trabajos que tienen como objeto macroestructuras no descartan sin embargo observaciones microsociológicas o estudios de casos ilustrativos;

- una variación de la integración más o menos equilibrada del pasado incorporado de los actores y de los aspectos contextuales o estructurales;

- una variación de las escalas de tiempo: la de la interacción de corta duración, la de la biografía de un individuo o la mucho mayor de la vida de grandes grupos o de grandes instituciones;

- y una variación del número de actores individuales estudiados, desde el individuo singular -por ejemplo Saint Louis, Flaubert, Mozart o completos desconocidos- al grupo de individuos (micro-grupos, miembros de una organización o de una institución, cuerpo profesional, categorías, clase o fracción de clase, comunidad, etc.).

Esta diversidad no constituye un defecto o un obstáculo; es sobre todo el signo de una gran riqueza y de una cierta vitalidad. Pero contribuye a tornar más difícilmente legibles los desafíos y los objetivos de estas ciencias. Hay sin embargo una unidad detrás de la multiplicidad de maneras de hacer sociología, historia o antropología.

La preocupación por esclarecer los problemas que impulsa mi práctica conduce a mostrar porqué el sociólogo no debería tener que "elegir su bando" especializándose en el estudio de un nivel de la realidad social o poniendo en práctica siempre la misma escala de observación del mundo social, sino que debería ajustar sus herramientas en función de los tipos de objetos que estudia (y que pueden variar) y de los problemas que pretende resolver.

Utilizando los conceptos de disposiciones y de contextos, no quisiera que se entienda, efectivamente, que éstos suponen privilegiar una escala de observación o un nivel de análisis de la realidad social particular. La práctica disposicionalista-contextualista puede inspirar tanto trabajos que se den por objetivo la comprensión de las variaciones -de las prácticas, de los comportamientos, de las actitudes- inter-sociedades o inter-épocas, como los que buscan dar cuenta de variaciones inter-grupos, inter-individuales (en el seno de grupos relativamente homogéneos) o intra-individuales, sincrónicos o diacrónicos, de un contexto de acción a otro. La misma práctica permite estudiar tanto individuos singulares, como interacciones entre algunos individuos, micro-grupos, instituciones o el espacio de las clases sociales. Las “disposiciones” pueden ser las de un único individuo, como las de algunos individuos en interacción o en inter-relación, en situaciones familiares, profesionales, lúdicas, escolares, públicas, etc., o las de una categoría o de un grupo de individuos que hayan vivido condiciones de socialización parcialmente similares (experiencias familiares $\underline{11}$, experiencias de género $\underline{12}$, experiencias en instituciones religiosas, deportivas, militares, políticas, etc.).

El "contexto” en sí puede ser tanto el espacio nacional de las clases y fracciones de clase, como un microcosmos social (“campo”, "juego”, “mundo”), una organización o una institución, un grupo o sub-grupo, y hasta un marco local de interacción.

Tal y como la concibo, una sociología disposicionalista-contextualista puede entonces interesarse por los mismos aspectos del mundo social y con el mismo afán por el detalle que una sociología interaccionista de tipo goffmaniano, pero introduciendo la idea según la cual los interactantes en presencia son portadores de disposiciones y competencias y que ponen en juego en la interacción, tanto para "definirla" como para actuar, 
esquemas de percepción, de apreciación, de interpretación, competencias retóricas o gestuales, maneras de ver, de sentir y de reaccionar que han constituido durante sus experiencias sociales (y en parte interaccionales) pasadas. Un interaccionismo disposicionalista, que fuese capaz de estudiar de cerca tanto las condiciones de desencadenamiento o de activación de las disposiciones como las de su inhibición o suspensión, debería también resaltar el hecho de que los actores están desigualmente preparados para los diferentes tipos de encuentros sociales a los cuales se enfrentan permanentemente y que son más o menos capaces, igualmente, de comportarse bien, de evitar meter la pata o de hacer torpezas. También están desigualmente predispuestos, por sus experiencias sociales pasadas, a adoptar protagonismo o roles secundarios, roles de liderazgo o de seguidores, etc.

En la gran mayoría de los casos, los investigadores en ciencias humanas y sociales definen su propia práctica procediendo por exclusiones; exclusiones:

de los productos de la historia cristalizada (vs. la acción en curso),

de las disposiciones (vs. las obligaciones contextuales de la acción),

de las estructuras macrosociológicas (vs. los encuadres microsociológicos),

del individuo (vs. los grupos), etc.

Posicionándose de este modo, presuponen que su punto de vista es mejor que el de otros, en teoría, independientemente del tipo de problema que busquen solucionar.

En efecto, existen múltiples maneras de poner a prueba el programa inscripto en una fórmula tal como:

$$
\text { "Disposiciones + Contextos = Prácticas” }
$$

Esta última puede engendrar trabajos muy distintos según la escala de observación puesta en práctica y el nivel de realidad social buscado. También se puede, dependiendo de los trabajos realizados, insistir más particularmente sobre tal o cual de sus componentes. En cada estudio particular, el investigador puede de este modo dirigir el esfuerzo de su investigación o bien hacia los contextos (sus estructuras, sus modalidades propias de funcionamiento, sus reglas de juego específicas, la naturaleza de lo que allí sucede, etc.) ya sean mundos, campos, juegos, instituciones, micro-grupos o encuadres restringidos de interacción, o bien hacia los actores y sus disposiciones y competencias (los productos de experiencias socializadoras sucesivas y/o paralelas por las cuales han pasado).

Y en ambos casos, puede insistir en el proceso de fabricación (sociogénesis) de los contextos o de los actores, o bien estudiar un estado, en un momento determinado, de un contexto o de un patrimonio (individual o colectivo) de disposiciones y competencias.

En relación a los contextos, el investigador puede hacer la historia de éstos, captar los mecanismos de funcionamiento en un momento dado o bien además estudiarlos bajo el ángulo de su rol socializador, observando y analizando la manera en la que la familia, la Iglesia, el Partido, el grupo de pares, la institución deportiva, el medio profesional, etc., contribuyen a formar a los actores que los frecuentan.

En relación a las disposiciones y a las competencias incorporadas, el mismo investigador puede hacer el análisis sociogenético de su formación en individuos o grupos determinados o estar más atento al modo en el que, una vez constituidos, son sutilmente movilizadas, desencadenadas, en la dinámica de las acciones e interacciones, en función de los contextos de la práctica, o a lo largo de un recorrido biográfico hecho de "elecciones" o de “decisiones” y a veces de bifurcaciones $\underline{13}$. Puede, por último, preguntarse cómo se transfieren o no de un contexto de formación hacia otros contextos. 
Mencioné anteriormente el carácter necesariamente imperfecto de las realizaciones empíricas que no pueden siempre buscar el equilibrio explicativo (e interpretativo) de la fórmula científica disposicionalista-contextualista. Pero las investigaciones no serían leídas ni utilizadas de la misma manera si se presentasen claramente como partes de un programa, como realizaciones parciales de un programa más completo, más que como suertes de fines en sí completas en su género (lo que, por la obligación que impone el juego de la competencia académica, es más frecuente).

Serían percibidas más como puntos de apoyo para construir objetos más amplios o más complejos que como vistas parciales que se las dan de vistas globales o totales y que desalientan la búsqueda de articulaciones o de combinaciones.

\section{Notas}

1 Traducción de Aimé Pansera. Maestranda en Sociología de la cultura y análisis cultural (IDAES-UNSAM).

$\underline{2}$ Cf. sobre todo L'Homme de vérité, Paris, Odile Jacob, 2002 y “Les bases neurales de l'habitus”, en G. Fussman (bajo la dir.), Croyance, raison et déraison, Paris, Odile Jacob, 2006, p.143-158.

$\underline{3}$ Y aquí también, tanto el entrenamiento intensivo como el detener el entrenamiento se objetivan en el cerebro (las redes neuronales). Cf. B. Draganski, C. Gaser, V. Busch et al., « Neuroplasticity : changes in grey matter induced by training ", Nature, 2004, n 427, p. 311-312 y B. Draganski, C. Gaser, G. Kempermann et al., "Temporal and spatial dynamics of brain structure changes during extensive Learning ", The Journal of Neuroscience, 2006, n² 26, p. 6314-6317.

4 D. Hume, Abrégé d'un livre récemment publié, intitulé : Traité de la nature humaine dans lequel le principal argument est plus amplement illustré et expliqué [1740], 2002. Web quebequense de los «Clásicos de las ciencias sociales » : http://www.uqac.uquebec.ca/zone30/Classiques des sciences sociales/index.html.

5 M. Mauss, "Relaciones reales y prácticas entre la psicología y la sociología”, Sociología y antropología, Madrid, Tecnos, 1971 (ed. francesa Paris, PUF, Quadrage, 1991, p.283-310).

$\underline{6}$ Por ejemplo, Luc Boltanski defendió, en algunos de sus textos, una visión exclusivamente contextualista de la acción, cuando dijo interesarse sobre todo por las “obligaciones” ligadas al "dispositivo de la situación en la cual están ubicadas las personas” [L. Boltanski, El Amor y la justicia como competencias: tres ensayos de sociología de la acción, Madrid, Amorrortu, 2000 (ed. francesa, Paris, Métailié, 1990, p.69)]. Otorga a los actores, por otra parte, “competencias” cuya sociogénesis (tanto histórica como individual) nunca es estudiada ("Consideramos que pertenece a la competencia de todos los miembros normales de una misma sociedad el poder aprehenderlas y tenerlas en cuenta.”, ídem).

$\underline{7}$ De este modo, escribe que "la estructura y la forma del comportamiento de un individuo dependen de la estructura de sus relaciones con los demás individuos” [N. Elias, La sociedad de los individuos, Barcelona, Edicions 62, 1990 (ed. francesa, Paris, Fayard, 1991, p.104)], o también que "las diferentes vías posibles entre las cuales él [el individuo] opta, le son prescritas por la constitución de su círculo de acción y sus relaciones de interdependencia" (ibid., p.95).

$\underline{8}$ Cf. B. Lahire, El Hombre plural. Los resortes de la acción, Barcelona, Bellaterra, 2004 (ed. francesa Paris, Nathan, Essais \& Recherches, 1998); Portraits sociologiques. Dispositions et variations individuelles, Paris, Nathan, Essais \& Recherches, 2002 y La Culture des individus. Dissonances culturelles et distinction de soi,, Paris, La Découverte, Laboratoire des sciences sociales, 2004.

9 P. Bourdieu, La distinción: Criterio y bases sociales del gusto. México, Taurus, 2002 (ed. francesa Paris, Minuit, 1979, p. 112). 
10 B. Lahire, L'Esprit sociologique, Buenos Aires, Manantial, 2006 (ed. francesa, Paris, La Découverte, Laboratoire des sciences sociales, 2005).

11 Cf. B. Lahire, Tableaux de familles. Heurs et malheurs scolaires en milieux populaires, Paris, Gallimard/Seuil, Hautes Études, 1995 así como el trabajo de G. Henri-Panabière, Des héritiers en "échec scolaire », Paris, La Dispute, 2010.

$\underline{12}$ Cf. C. Mennesson, Etre une femme dans le monde des hommes. Socialisation sportive et construction du genre, Paris, L’Harmattan, 2005 y M. Court, Corps de filles, corps de garçons : une construction sociale, Paris, La Dispute, 2010.

13 Cf. S. Denave, Ruptures professionnelles. Processus, contextes et dispositions, tesis de doctorado de sociología, Universidad Lumière Lyon 2, 24 de noviembre de 2008.

\section{Bibliografía}

Boltanski, L. (2000). El Amor y la justicia como competencias: tres ensayos de sociología de la acción. Madrid: Amorrortu (ed. francesa, Paris, Métailié, 1990, p.69).

Bourdieu, P. (2002). La distinción: Criterio y bases sociales del gusto. México: Taurus, (ed. francesa Paris, Minuit, 1979, p. 112).

Changues, J.P. (2002): L’Homme de vérité. Paris: Edition Odile Jacob.

Court, M. (2010). Corps de filles, corps de garçons: une construction sociale. Paris: La Dispute.

Denave, S. (2008). Ruptures professionnelles. Processus, contextes et dispositions, tesis de doctorado de sociología, Universidad Lumière Lyon 2, 24 de noviembre de 2008.

Draganski, B.; Gaser, C.; Busch, V. et al. (2004). Neuroplasticity : changes in grey matter induced by training, Nature, $n^{\circ} 427$, p. 311-312.

Draganski, B.; Gaser, C., Kempermann, G. et al. (2006). Temporal and spatial dynamics of brain structure changes during extensive Learning, The Journal of Neuroscience, ${ }^{\circ}$ 26, p. 6314-6317.

Elias, N. (1990). La sociedad de los individuos. Barcelona: Edicions 62 (ed. francesa, Paris, Fayard, 1991, p.104).

Fussman, G. (2006). Croyance, raison et déraison. Paris: Editions Odile Jacob.

Gauss, M. (1971). Sociología y antropología. Madrid: Tecnos (ed. francesa Paris, PUF, Quadrage, 1991, p.283310).

Henri-Panabière, G. (2010). Des héritiers en « échec scolaire ». Paris: La Dispute.

Hume, D. [1740], 2002. Traité de la nature humaine. Web quebequense de los « Clásicos de las ciencias sociales» http://www.uqac.uquebec.ca/zone30/Classiques des sciences sociales/index.html.

Lahire, B. (1195).Tableaux de familles. Heurs et malheurs scolaires en milieux populaires. Paris: Gallimard/Seuil, Hautes Études.

Lahire. B. (2004). El Hombre plural. Los resortes de la acción. Barcelona: Bellaterra (ed. francesa Paris, Nathan, Essais \& Recherches, 1998).

Mennesson, C. (2005). Etre une femme dans le monde des hommes. Socialisation sportive et construction du genre. Paris: L’Harmattan. 